\title{
Algunas líneas de investigación en gestión del conocimiento jurídico: web semántica, ODR y derecho relacional
}

\author{
Some research lines in legal knowledge management: semantic web, ODR and relational law
}

\author{
Pompeu Casanovas \\ Instituto de Derecho y Tecnología (IDT), Universidad Autónoma de Barcelona (España) \\ pompeu.casanovas@uab.cat
}

\begin{abstract}
Resumen
En este artículo se plantean algunas de las lecciones aprendidas en la elaboración de ontologías jurídicas y sistemas para la web semántica, con especial atención a la creciente participación de los usuarios en la red. El artículo contiene una breve descripción de los modelos de informática jurídica documental de Akoma Ntoso y MetaLex. Un tercer modelo lo constituyen las formas de Online Dispute Resolution (ODR), tecnologías del acuerdo y mediación en línea que permiten plantear también el desarrollo de formas de derecho relacional. Los proyectos del Instituto de Derecho y Tecnología (UAB-IDT) siguen esta última vía.
\end{abstract}

Palabras clave: Informática jurídica. Gestión del conocimiento jurídico. Crowdsourcing. Ontologías jurídicas. Web Semántica. Akoma Ntoso. MetaLex. Resolución de Disputas en Línea. Derecho relacional.

\section{Introducción}

En este artículo se plantean algunas ideas desarrolladas en proyectos de investigación por el Instituto de Derecho y Tecnología de la UAB en estos últimos años (http://idt.uab.cat ). En 2001, justo después que Tim Berners-Lee lanzara la idea de la web semántica por primera vez, R.V. Benjamins, J. Contreras, M. Poblet y yo mismo empezamos a trabajar en esta línea (Benjamins, Casanovas, Breuker y Gangemi, 2005). En este momento, ya había diversas ontologías jurídicas nucleares, realizadas desde 1995 en varios Proyectos Europeos del V y VI Programas Marco por los equipos, entre otros, de Joost Breuker, Aldo Gangemi y André Valente. Hemos vertido recientemente algunos artículos seminales de esta época al castellano (Vallbé, Fernández-Barrera, Casellas y Casanovas, 2012), así como el Manual de Web Semántica del MIT (Antoniou y van Harmelen, 2008).

El trabajo ontológico había empezado, pues, antes y no después de que se tuviera la idea general de estructurar el contenido de la Web a partir del famoso pastel de capas de la Web

\begin{abstract}
I refer in this paper some of the learned lessons in the construction of legal ontologies and systems for the Semantic Web, with a special eye on the increasing end-user participation on the web. The Akoma Ntoso and MetaLex models of legal information documentation are briefly described. It is argued that, in addition to these models, Online Dispute Resolution (ODR) forms, agreement technologies and online mediation foster the development of what it may be defined as relational law. The latter is the research line followed by the projects carried out at the UAB Institute of Law and Technlology.
\end{abstract}

Keywords: Legal informatics. Legal knowledge management. Crowdsourcing. Legal ontologies. Semantic Web. Akoma Ntoso. MetaLex. Online Dispute Resolution (ODR). Relational law.

Semántica de Tim Berners-Lee. También son anteriores los Institutos de Información Jurídica de Tom Bruce (2000, 2009), Graham Greenleaf (2010) y Daniel Poulin (2009) (http://www.world lii.org/); y la actividad lingüística de procesamiento de lenguaje natural. Igual sucede con la construcción de Wordnet (2006, en inglés, en castellano y en catalán) y el tratamiento de los conceptos del sentido común al que tanto tiempo dedicaran Buck Buchanan y Edward Feingenbaum. CommonKADS (por Knowledge Acquisition and Documentation Structure) fue uno de los proyectos pioneros de ingeniería del conocimiento que aplicó las lecciones aprendidas en la gestión de las primeras bases de datos y de los sistemas expertos de los años setenta y ochenta (http://www.commonkads.uva.nl/ ) (Schreiber, Akkermans, Anjewierden, de Hoog, Shadbolt, van de Velde y Wielinga, 2000).

Creo que, se mire por donde se mire, los logros de la informática jurídica en el tratamiento de textos proceden no de la perspectiva de la Web Semántica (WS), sino del período anterior. Si esto es así, ¿qué podemos considerar pues que ha aportado esta visión? ¿Dónde están las debi- 
lidades de la Web Semántica, y dónde sus aportaciones?

Voy a dividir mi contribución en cuatro partes. En primer lugar, distinguiré la segunda generación de aplicaciones de WS de sus orígenes e introduciré la idea de Web de Servicios Semánticos; en segundo lugar, voy a referirme a dos estrategias distintas de informática jurídica documental para la WS, representadas por los equipos de Amsterdam y de Bolonia; en tercer lugar relataré algunas de las lecciones aprendidas durante estos años en el trabajo del IDT. Dejaré para el final la idea de crowdsourcing y la participación de usuarios de la red en la regulación de la misma. Es lo que voy a denominar, a falta de un término mejor, derecho relacional.

\section{La segunda generación de la Web Semántica}

\subsection{Tres modalidades de la misma red}

La Web Semántica nació como una idea para la configuración del contenido de la red como conocimiento estructurado. Se desarrolló rápidamente como una serie de lenguajes apilados de anotación. Su segunda versión, sin embargo, ha insistido no tanto en la operatividad de una estructura lógicamente articulada, sino en su orientación. No se trata ahora de lograr la organización del conocimiento de una forma completamente desarrollada, sino de conseguir rapidez, eficacia y seguridad en su uso como gestión del conocimiento compartido por el usuario. Se produce así una polarización entre la orientación pragmática del usuario final de los sistemas de gestión, y la orientación semántica colectiva de millones de usuarios implicados. Se concibe, en efecto, como una Red de Datos [Web of Data] y ya no de páginas, donde el link es interno a cada sitio, con una relación de objeto a objeto. Una manera simple de expresarlo sería la siguiente:

- Web 1.0: Internet (hipervínculos)

- Web 2.0: Expresividad (Tags, modelo del prosumer: consumidor y generador de contenidos): Flikr, YouTube, Wikipedia, Facebook...

- Web 3.0: Expresividad + Semántica (web de objetos vinculados -"linkados"- y no solo de páginas Web)

Red, red social, red de datos. La relación entre la Web 1.0 y la web 2.0 puede permitirme, por ejemplo, descubrir el texto y la música de un fox-trott italiano de 1937 titulado Vivere a partir de una sola frase conocida -la mia bella donna se n'è andata. Este único conocimiento puede proporcionarme mucha más información, además del título, el autor (Carlo Andrea Bixio) y el texto completo de la canción (Oggi che magnifica giornata, che giornata di felicità, la mia bella donna se n'è andata, m'ha lasciato al fine in libertà...). Si alguien la ha subido a You Tube, como es el caso, puedo escuchar la grabación original de Aldo Visconti y la versión que realizó mucho más tarde Luciano Pavarotti (http://www .youtube.com/watch? $v=x Y t D t c w 4 J b w)$.

Hace algunos años imaginábamos poder silbar una melodía o recitar un texto - - , en este caso, un texto aproximado en italiano, o una traducción al castellano de la letra- y que el sistema reconociera la canción y devolviera la información solicitada. Éste es el sueño de la WS: lograr una relación tal entre el usuario y el sistema de búsqueda que éste pueda comprender la motivación del primero y las características de la información demandada a partir solamente del contenido semántico de la misma.

Bien. Ahora existen ya aplicaciones para móvil que realizan esta función de recuperación de información a partir de la melodía (http://www. soundhound.com). Sólo que no se han construido a través de la WS, sino mediante reconocimiento de patrones. La semántica no ha sido necesaria para lograrlo.

Sin embargo, la cosa no acaba aquí. La versión original de la canción que se encuentra en You Tube se acompaña de un vídeo con carteles originales de los años treinta con coches antiguos FIAT de época. Esto, desde el año 2008, ha dado lugar a diversos comentarios en cascada sobre el fascismo, e. g.: Ora è diventata F:E.A.T, fabbricazione ebraica automobili torino con gli ELKANN. Y a réplicas y contraréplicas:

- Non ti vergogni ad essere in vita e ad avere un cervello che si alimenta creando poi questi malsani pensieri?

- La madre dei deficenti é sempre incinta... la storia insegna purtroppo

Puedo encontrar fácilmente, en suma, mucha más información de la que en principio yo andaba buscando; y la verdad es que, aunque parece que no sé muy bien lo que hacer con ella, es a partir de esta información adicional y de las búsquedas complementarias que realizo por curiosidad como reconstruyo a partir de fragmentos de información un discurso más o menos coherente sobre la canción originalmente demandada.

Este modo de proceder - algo errático, guiado por la curiosidad, pero que acaba produciendo 
resultados medianamente satisfactorios - fue observado desde el principio por los investigadores, y asumido como parte de los patrones de búsqueda del usuario en la red. Abre muchas posibilidades también desde el punto de vista técnico. Por ejemplo, se puede reconstruir el itinerario personal del usuario, pueden seguirse los logs a partir de la trazabilidad, etc. La otra cara de la moneda es que esto implica tener algo de tiempo para perder en la búsqueda: no puede darse por descontado en el comportamiento actual de los usuarios.

\subsection{Web Semántica}

Hasta ahora no he hablado de semántica. En los sistemas de búsqueda (information retrieval), la semántica ha tenido un papel limitado, quizás porque lo que quiere obtenerse es un resultado inmediato y detenerse en la estructura del contenido ralentiza la obtención de este resultado. Ricardo Baeza-Yates (2009) lo ha vuelto a decir recientemente. La premisa es que la gente no quiere buscar. Ya no se trata exactamente de la WS inicial, sino de encontrar la relación implícita que hay entre los objetos en el uso de la red. Hay que procurar establecer el vínculo existente entre metadatos explícitos e implícitos en la red, explotando todo el potencial de la misma.

Esta misma evolución hacia la relación entre conocimiento estructurado y usuario es lo que ha llevado al equipo de Enrico Motta, en la Open University (e.g. D’Aquin, Motta, Sabou, Gridinoc, López y Guidi, 2008), a formular la idea de concebir las aplicaciones de la segunda generación de WS en términos de personalización del conocimiento y de explotación de todo el conocimiento diseminado en la red:

- reutilización (vs. generación de datos semánticos)

- sistemas de multi-ontologías (vs. sistemas de ontología única)

- apertura a recursos semánticos de alto nivel [top semantic resources]

- escalabilidad (tan importante como la calidad de los datos)

- apertura a todos los recursos de la red (también los no-semánticos)

- adaptación al paradigma de la Web 2.0

- apertura a la web de servicios

Desde hace ya algún tiempo, como muestra el gráfico de la figura 1 , los servicios semánticos, las aplicaciones y la interfaz de usuario se sitúan ya en el vértice de la WS, algo que no es- taba contemplado en el diseño original. Ahora el esfuerzo tiende a concentrarse más en los sistemas de consulta (SPARQL) y en los de definición e intercambio de reglas (RIF/SWRL), y menos en OWL. La figura 2, más reciente, representa el intento de Tim Davies (2011) de reelaborar las distintas partes relevantes de la WS como "datos vinculados", Linked Open Data. Es un hilo que retomaré más tarde.

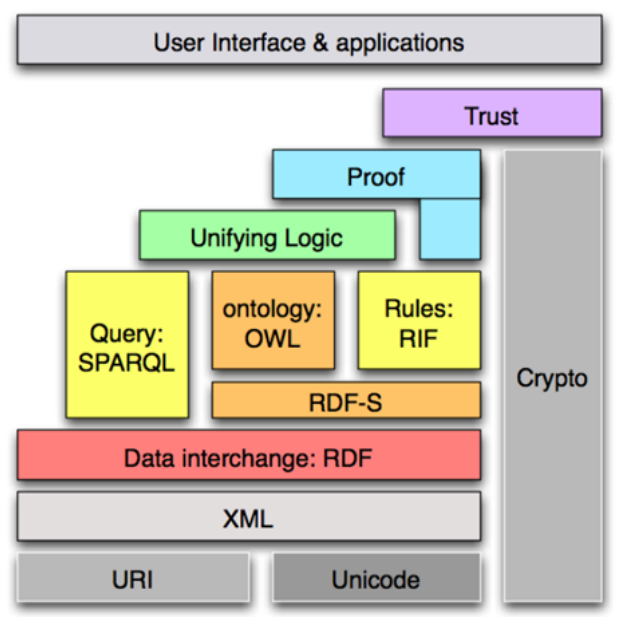

Figura 1. Versión de la pila de lenguajes de la WS (Bratt, 2006)

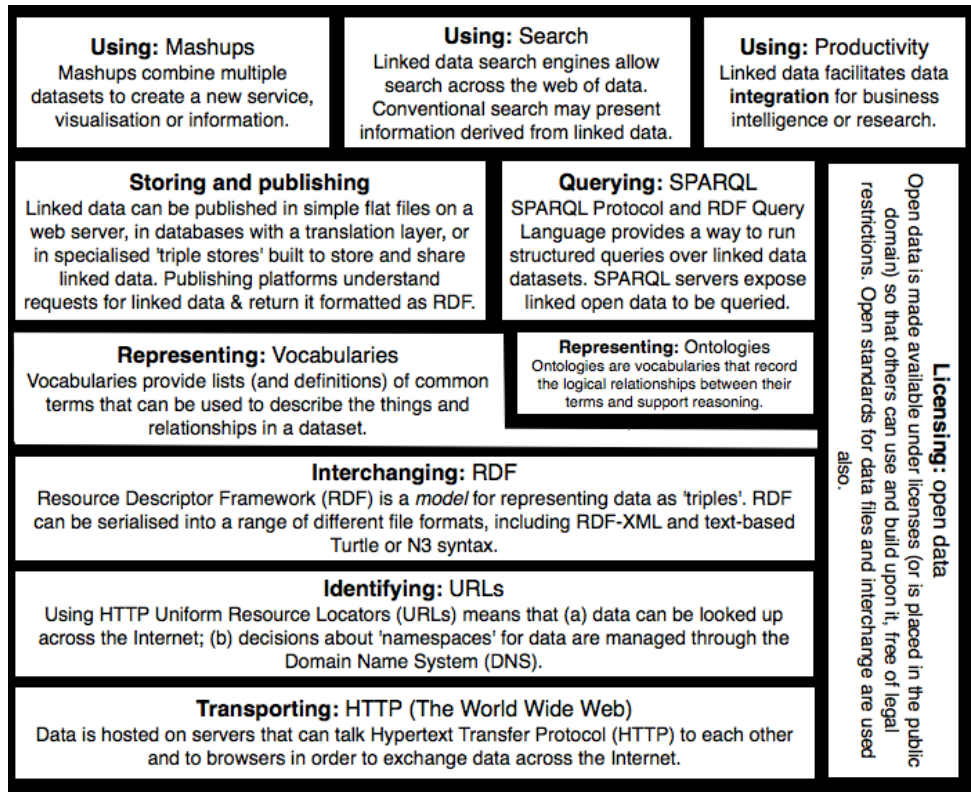

Figura 2. Elementos de Linked Open Data Stack (LODS) (Tim Davies, 2011)

\subsection{Servicios Web Semánticos (SWS)}

Los servicios web semánticos (SWS) son, pues, un componente de la WS, el servidor final de un sistema de servidor-cliente para la interacción 
automática entre máquinas vía WWW (http://www.wsmo.org). Debo aclarar que a veces utilizo esta expresión (SWS) referida a los servicios web jurídicos con el significado de "servicios web jurídicos con asistencia semántica".

Pero incluso con este tipo de escenarios, el desarrollo de la Web Semántica parece seguir el gráfico de Gartner para las tecnologías emergentes, después de situarse en la "fase de desilusión" que sigue al pico de expectativas "exageradas" o excesivas de la primera etapa, en 2005. De hecho, las tecnologías de la WS no aparecen en el top 10 del ranking del 2012, y así como Gartner parece apoyar a las tecnologías colaborativas de la Web 2.0, como ha hecho notar Jon Brodsky (2007), las de la 3.0 no merecen su atención. Se trataría de una "comercialización táctica" (marketing ploy).

Contra ello ha reaccionado Novak Spivak, quien ha recordado que estas denominaciones periodísticas no se refieren a la técnica, sino a la disponibilidad de su uso en el mercado. Sucede justamente al revés: la Web 3.0 se compone en realidad de tecnologías emergentes, no así la web 2.0. E. g., Tagging no es una tecnología, sino un patrón de diseño. Igual que AJAX (Asynchronous JavaScript and $X M L$ ) resulta de una combinación de tecnologías ya existentes. Desde esta perspectiva, la innovación en la red proviene de la capacidad de reinventar la WS para potenciar y mejorar las aplicaciones, el funcionamiento de los servicios y la interoperabilidad entre los sistemas, y entre éstos y los interfaces de usuario (Spivak, ibid. Énfasis añadido):

Esencialmente, la Web Semántica facilita la transformación gradual de la red en una base de datos. Se trata de un cambio estructural en profundidad que finalmente va a afectar a todas las capas de la tecnología web.

La misma orientación tiene la Internet de Servicios basados en los pilares de WS de John Davies et al. (2009) y en los Servicios Web basados en datos vinculados de Carlos Pedrinaci y John Domingue (2010). En este modelo, las comunidades de las redes sociales quedan integradas en el desarrollo de la tecnología para la interoperabilidad de los servicios. Creo que la posición de Spivak, Davies, Pedrinaci y Domingue tienen mucho sentido. $Y$ de hecho es lo que venimos siguiendo desde hace ya algunos años en los programas de investigación en WS y Derecho del IDT-UAB. Pero antes de exponer nuestra estrategia, conviene mencionar dos aproximaciones más, ya muy establecidas, relativas a WS y Derecho que compiten (y cooperan) entre sí por lograr la categoría de estándar europeo. Podríamos denominarlas respectivamente la perspectiva de Bolonia, y la perspectiva de Amsterdam. Akoma Ntoso (http://www.ako mantoso.org/), por una parte, MetaLex (http:// www.metalex.eu/ ), de otra.

\section{Aproximaciones jurídicas a la Web Semántica}

\subsection{Akoma Ntoso y MetaLex}

Akoma Ntoso ("corazones unidos") constituye un conjunto de estándares en XML automáticamente legibles para el intercambio y reusabilidad de documentos judiciales, legislativos y parlamentarios. Es decir, se sitúa al nivel descriptivo de los distintos niveles de los documentos oficiales para su clasificación, búsqueda y uso posteriores. MetaLex busca esencialmente lo mismo. Uno empezó como un programa para el análisis y clasificación de los documentos en los Parlamentos africanos. El segundo, que es anterior al primero, como un conjunto de estándares para la descripción de las leyes holandesas. Ambos conjuntos coincidieron en el proyecto europeo ESTRELLA (2007-2009) (http://www. estrellaproject.org) y recogieron experiencias previas como la danesa LexDania y la italiana Norma-in-Rete.

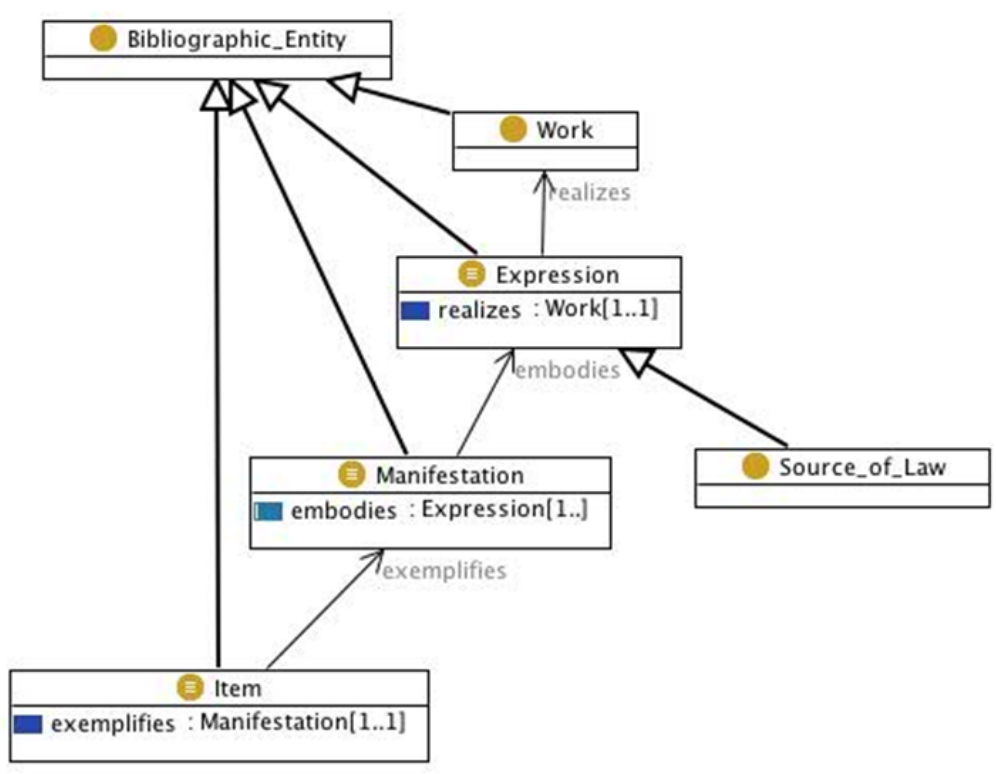

Fig. 3. Jerarquía de entidades bibliográficas en META-LEX (CEN MetaLex Workshop Agreement, 2006)

No voy a compararlos aquí, aunque sería ciertamente muy interesante y es un trabajo que es aconsejable realizar en el futuro de forma sistemática. Hay notables diferencias en la orienta- 
ción, metodología, conceptualización y desarrollo, a pesar de los esfuerzos realizados para lograr la interoperabilidad en los estándares (CEN-MetaLex Workshop, 2006, 2008, http://www.cen.eu) (Fig. 3).

\section{Como señala Hoekstra $(2011$, p. 7),}

CEN MetaLex es un estándar XML extrajurisdiccional para representar, publicar e intercambiar la estructura de los recursos jurídicos. [...] Los elementos de MetaLex son puramente estructurales. Los elementos sintácticos (estructura) al especificar para cada elemento su nombre y su modelo de contenido. Lo que esto implica es esencialmente preparar el camino para una descripción puramente semántica de los tipos de contenido de los elementos en un documento XML.

Precisan Barabucci, Cervone, Palmirani, Peroni y Vitali (2010, p. 134):

La función de Akoma Ntoso es marcar los textos legislativos y jurídicos de modo que el conocimiento jurídico y la estructura jurídica del texto pueda ser comprendida por aplicaciones informáticas presentes y futuras. Esto significa que los textos jurídicos forman la base sobre la que existen los documentos de Akoma Ntoso. Éstos añaden información por encima del texto (decoran el texto en el la jerga del marcado automático). La información añadida puede ser contemplada en si misma como compuesta por distintas capas estratificadas (texto, estructura, metadatos, etc.

Las diferencias se perciben mejor en los desarrollos ontológicos, más allá de la base comúnmente aceptada, LKIF (Legal Knowledge Interchange Format) para MetaLex (Breuker et al. 2007; Boer et al. 2008; Boer, 2009 ); LIDO (Legal Informatics Document Ontology) para Akoma Ntoso (Palmirani et al., 2011).

Akoma Ntoso está más cerca del texto, a partir de una concepción normativa que tiene muy presente la teoría jurídica (Barabucci et al. 2010). MetaLex-LKIF, en cambio, se desarrolla a partir de modelos epistemológicos más basados en las ciencias cognitivas que en los sistemas normativos (Boer, 2009; Breuker y Hoekstra, 2010); y ello tiene consecuencias importantes tanto para la programación en XML y RDF como para las ontologías nucleares utilizadas en los esquemas de razonamiento en OWL.

MetaLex-LKIF, por ejemplo, considera las leyes parlamentarias como un aspecto intencional del hecho de producción de una norma: el texto es así contemplado como un resultado (a veces sub-óptimo) en relación a las acciones intencionales. Alguna vez el texto final ha sido incluso referido como residual respecto a la acción; sin embargo, la modelización se realiza solamente a partir de los textos resultantes (Boer, 2009, p. 165):
MetaLex organiza los metadatos alrededor de ocurrencias -eventos y acciones. Para metadatos esto no es lo común, pero en efecto se trata de lo mismo que hemos venido realizando hasta el momento con la interpretación del objeto de las normas jurídicas: éstas se aplican a acciones, o al resultado de acciones.

La perspectiva de Bolonia no posee ni desea poseer este trasfondo marcadamente cognitivo y de psicología social. Hay nociones que me parecen clave en uno y otro desarrollos, e.g. la noción de "concepto dinámico" en MetaLex, y el de "bloque de enmiendas" en Akoma Ntoso. La relación con la base cognitiva se realiza en el primer caso de forma directa, fundacional. En cambio, en el segundo caso, Akoma Ntoso se vincula a la cognición (causalidad, intencionalidad, acción) a través de la teoría jurídica (y la perspectiva sobre la web) desarrollada por Giovanni Sartor (2005-2011), y -en lógica- por el mismo Sartor, Nino Rotolo y Guido Governatori. Esto enlaza con el interés por los sistemas multiagentes, y con el desarrollo de XML jurídico que llevan a cabo Fabio Vitali, Monica Palmirani $y$, en el ITTIG de Florencia, también con matices propios, el equipo de Daniela Tiscornia y Enrico Francesconi.

Cabe apuntar la actitud ligeramente distinta de ambos conjuntos de estándares en relación a la WS, pese al uso y desarrollo continuado de ontologías con metodologías de construcción similares. Ambos tienen una base sintáctica estructural y mantienen una ontología nuclear. Tienen asimismo una expresa vocación de ofrecer servicios jurídicos web, y una variedad de instrumentos para las funciones jurídicas básicas: legística (drafting), producción y clasificación de sentencias (sentencing), y mecanismos para la autoregulación contractual (contracting). Pero a pesar de la orientación de gestión de conocimiento jurídico en uno y otro caso, cabe notar que Akoma Ntoso pretende sostener la "web semántica jurídica", reflejando en ella su base normativa: "la visión de 'una sola web' [the 'one web' vision] puede ser traducida a la visión de 'una sola web jurídica' ['one legal-web' vision]" (Sartor, 2011, p. 17). MetaLex, en cambio, mantiene las distancias (Boer, 2009, 262; también Hoekstra, 2011, 8):

Los estándares XML mediante los que se construye la web semántica tienden a identificar documentos en el nivel del ítem y su manifestación, mientras que el ámbito jurídico identifica documentos en el nivel de la expresión (versiones consolidadas) y de trabajo. Para la representación del conocimiento lo que interesa es el nivel de expresión puesto que es el nivel en el que los documentos son identificados y singularizados a partir de sus contenidos de información. 
El monismo jurídico de Akoma Ntoso contrasta con el pluralismo de MetaLex. Y, sin embargo, ambos asumen el principio básico de escritura administrativa: el soporte base es siempre un documento que se considera jurídico, es decir, validado por alguna autoridad competente encargada de su calificación como tal, o administrativo (en caso de no consistir en textos legislativos o judiciales). La validación de ambos estándares, pues, consiste en el reto de la traducción del HTML legislativo en que se presentan los documentos jurídicos oficiales a los estándares propuestos de XML y XML-schema.

\subsection{El enfoque del IDT}

Sin duda, el enfoque de Akoma Ntoso y el de MetaLex son esenciales para el desarrollo del Derecho en la red. Sin embargo, puesto que el CEN Workshop sólo se ocupa de un estándar $\mathrm{XML}$, las definiciones de Derecho que no tienen una contrapartida en la dimensión de información textual no son consideradas relevantes. Es cierto que el enfoque de MetaLex tiene en consideración el contexto y los elementos de gobernabilidad, espacialidad (la geo-localización de la legislación aplicable) y las prácticas de la e-administración (Boer y van Engers, 2010), pero siempre dentro de la expresión escrita de la legalidad, la jurisprudencia y la administración.

En el IDT proponemos una perspectiva conceptual complementaria, centrada en los datos y situaciones complejas de uso socialmente relevantes. Consideramos, e.g., que los mimbres con que se urde el derecho contemporáneo no solamente comprenden la dimensión textual, sino también otros soportes distintos al texto de importancia creciente, como la imagen o la oralidad misma. Así, los elementos de negociación, resolución y gestión de conflictos entran también como un objeto a tener en consideración en las redes sociales, en el comportamiento del usuario, y en la multiplicidad de actos que éste puede realizar a través de la red (Casanovas y Poblet, 2008, 2009a, 2009b).

Los desarrolladores de la WS se han dado asimismo cuenta de ello, puesto que las contradicciones e inconsistencias se producen ya al nivel de los metadatos y de las ontologías, y hay que prever mecanismos para la regulación manual, semi-automática o automática de estas situaciones. "La noción de confianza [trust] resulta esencial aquí, para dar soporte a las aplicaciones en la selección de recursos y ontologías compatibles con su punto de vista y entre sí" (D'Aquin et al. 2008, p. 27).
Hay tres puntos que unen todos los proyectos del IDT:

- la atención al usuario final, que constituye el punto de partida y llegada del proceso de investigación;

- la elaboración de datos (que pueden ser extraídos con los métodos tradicionales de las ciencias sociales) y la atención a su clasificación, análisis y tratamiento posterior;

- la construcción de prototipos, con ontologías que pueden incardinarse en escenarios concretos y que constituyen microsistemas, con reglas y procedimientos propios de actuación.

Es decir: el punto de partida jurídico no se da por descontado - como es el caso en Akoma Ntoso y MetaLex - sino que se construye de una forma un poco más amplia, al mismo tiempo que se diseña como una dimensión o parte de un sistema regulatorio que tiene un alcance más general, donde el almacenamiento, archivo, clasificación, tratamiento y posterior búsqueda de documentos no constituye la finalidad básica del sistema. Hemos denominado enfoque sociojurídico a esta perspectiva de construcción y uso de ontologías jurídicas (Casanovas, Casellas y Vallbé, 2011).

En realidad, soy consciente de que lo que estoy diciendo supone una vuelta a la inteligencia artificial aplicada a los viejos sistemas expertos. $Y$, de hecho, los problemas a los que nos enfrentamos siguen siendo en parte los mismos. Fundamentalmente, también tres: (i) la adquisición de conocimiento - el knowldege acquisition bottleneck de Feigenbaum-; (ii) la representación del conocimiento; (iii) y el diseño de arquitecturas flexibles del sistema para albergar los buses de servicios y realizar las funcionalidades y aplicaciones previamente diseñadas.

A ello cabe añadir los problemas de mantenimiento de las ontologías, los de interoperabilidad de sistemas y los derivados de la interrelación entre los usuarios en la comunidad. Voy a poner algunos ejemplos concretos, que corresponden a lecciones aprendidas. Quizás la más importante es que si se trabaja apegado a los usuarios e instituciones el ciclo de vida de los sistemas, prototipos y ontología absorben las características, pero también las limitaciones, de éstos.

luriservice, e.g. es un e-FAQ que modeló el conocimiento práctico de los jueces en su primer destino, diseñado para servir de apoyo a los jueces egresados de la Escuela Judicial Española. Tuvo una cuidadosa construcción 
etnográfica, elaborada a partir de diversas encuestas, y dos campañas sobre el terreno en más de 100 tribunales repartidos por todo el territorio nacional. Se construyeron varios prototipos, y diversas ontologías a partir de las preguntas formuladas por los mismos jueces en situación de guardia. El conocimiento modelado no estaba cubierto por ningún documento jurídico, sino que constituía un conjunto de soluciones prácticas que pasaban de generación en generación de magistrados. El sistema contenía unas 800 preguntas con sus correspondientes respuestas, escritas por los magistrados de la Escuela Judicial, y permitía también la búsqueda de sentencias asociadas (Fig. 4). El ciclo de construcción y validación de luriservice ha sido descrito en bastantes artículos de investigación. Una visión completa integrada de la construcción de las distintas ontologías se encuentra en Casellas (2011), y una buena descripción de los análisis de datos y pruebas con usuarios en Vallbé (2009).
El sistema fue presentado en 2006 oficialmente al CGPJ como resultado del Proyecto Europeo SEKT (2003-2006) (http://www.sekt-project. $\mathrm{com} /$ ). Sin embargo, nunca fue implementado en la red de la Escuela, pese a la buena voluntad de todos los implicados. Las razones son múltiples -aparición de legislación nueva relevante, e.g. sobre violencia doméstica; cambios internos en la propia Escuela; inexistencia de una intranet compatible del CGPJ; proceso de cambio de los sistemas informáticos del propio CGPJ, etc.- pero tienden a apuntar todos a que los ciclos institucionales en Derecho son a la vez más lentos y más rápidos que la evolución de la propia tecnología. Más lentos porque el proceso interno administrativo tiene su propia dinámica política y social. Más rápidos porque las situaciones jurídicas pueden cambiar radicalmente de un día a otro en función de la aparición de nuevas situaciones no reguladas o de legislación nueva, y ello afecta al contenido estructurado (Casanovas et al., 2011).

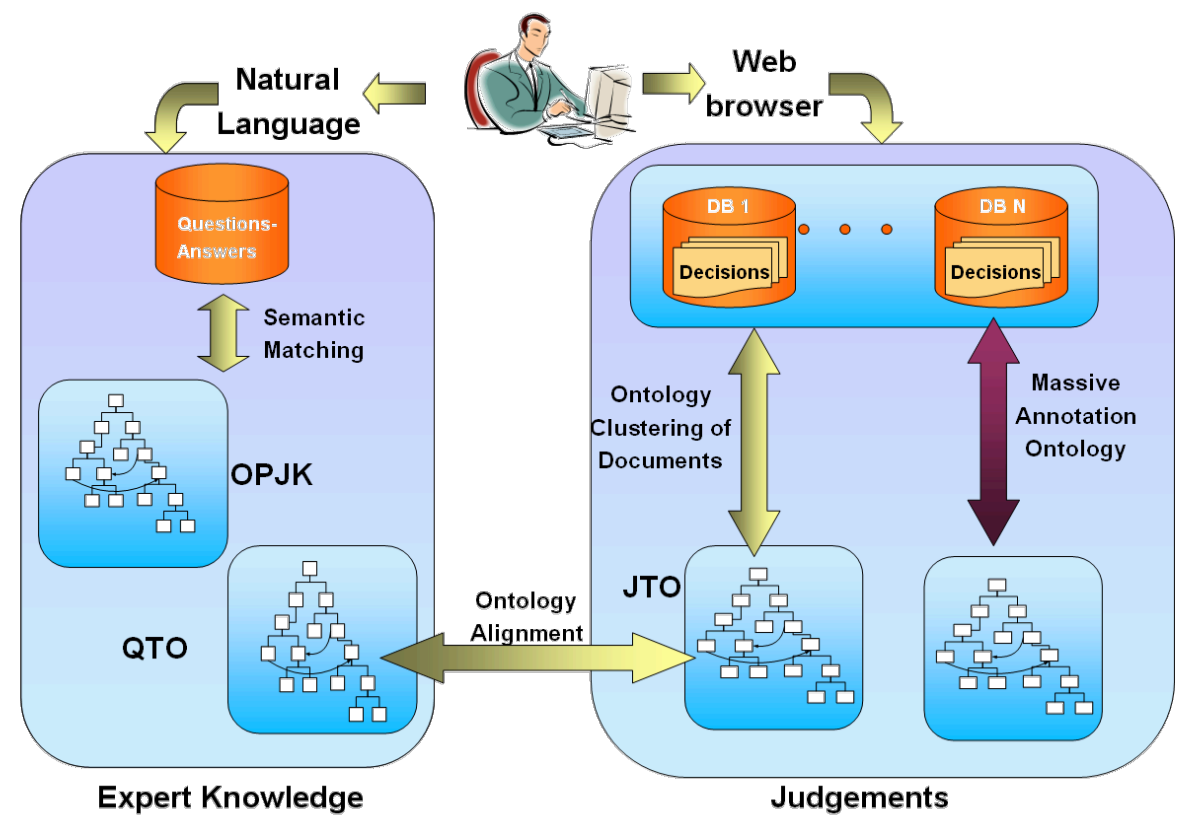

Fig. 4. Arquitectura original de luriservice (SEKT, 2006; Núria Casellas, 2011)

El ciclo iterativo para la generación e implementación de prototipos, modelos y ontologías, pues, tiene características propias en escenarios institucionales y jurídicos. Esto significa que deben imaginarse los procesos de construcción de sistemas basados en este tipo de aproximación sociojurídica teniendo en cuenta a la vez la vulnerabilidad y fragilidad de los microsistemas sociales y políticos. A pesar de ello, la experiencia adquirida nos ha permitido perfilar los métodos de la adquisición y validación de conocimiento jurídico, y un mejor planteamiento en el modelado del mismo para proyectos posteriores. Probablemente volveremos en el futuro a adaptar la misma idea a los contextos creados por la Web 2.0 y 3.0 .

Un segundo ejemplo viene constituido por la experiencia del IDT con los materiales audiovisuales (Bourcier, Casanovas, Dulong de Rosnay y Maracke, 2010). E-SENTENCIAS constituyó un proyecto para la clasificación e indexación automática de los videos producidos en los tri- 
bunales civiles, a partir de la Ley de 7 de Enero 2001/1.

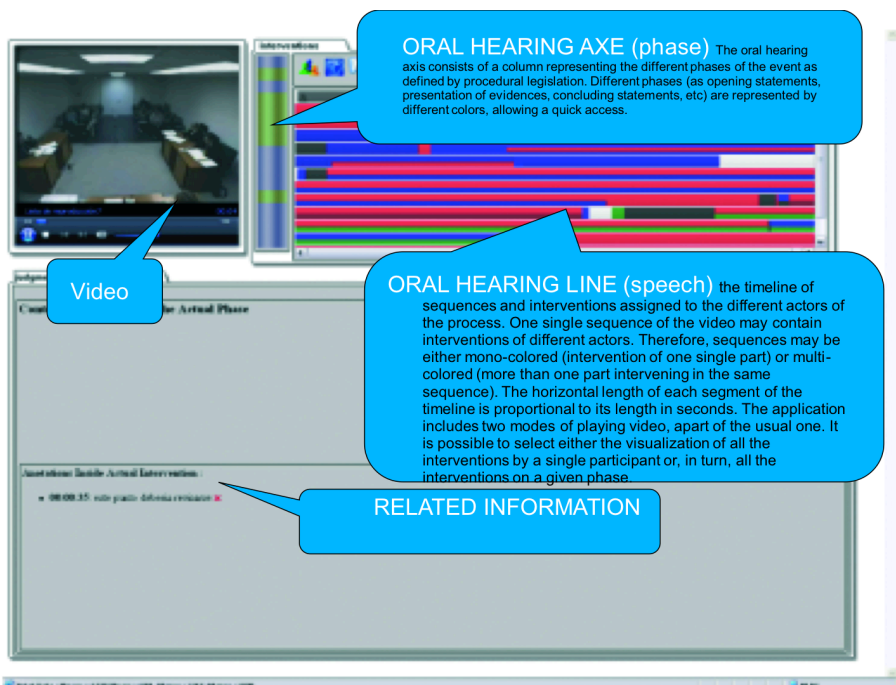

Fig. 5. Instrumento de navegación de E-sentencias, según un diseño de Xavier Binefa y Ciro Gracia.

(Casanovas et al., 2009)

Modelamos 8 procesos civiles en función de sus fases procesales reales -en los tribunales, no en la Ley de Enjuiciamiento Civil o en la doctrina. Pero tuvimos que recurrir a la diarización (fragmentación) del sonido para la segmentación de los videos: las ontologías no podían ser directamente extraídas de las imágenes. Esto, como es sabido, constituye un problema por sí mismo. Extraer conocimiento además de la secuencia de imágenes jurídicas para la elaboración ontológica presenta el problema de su simplicidad extrema: una cámara fija, con actores prácticamente fijos también, con pobreza de movimiento y de calidad en las imágenes, donde lo que cuenta es la exoforicidad del discurso, es decir, el contenido semántico de lo que se pronuncia en referencia a documentos o situaciones previas que se evocan en sala.

Lo importante, más que el documento audiovisual en sí que cuenta como prueba, deriva del cruce entre la línea de las intervenciones orales y la del procedimiento (sus fases o momentos procesales). Sólo la adopción de los dos ejes permitió la elaboración del instrumento de anotación y de navegación. Las ontologías, pues, se limitaron a reflejar la terminología típica en las fases. El resultado producido fue una ontología procesal, más que la ontología multimedia inicialmente prevista (Casanovas, Binefa, gracia, Teodoro, Galera, Blázquez, Poblet, Carrabina, Montón, Montero, Serrano y López-Cobo, 2009).

\section{Diálogo, ODR y derecho relacional}

\subsection{Crowdsourcing, sistemas regulatorios e} instituciones electrónicas

Desde hace algún tiempo, y sin que corresponda a una decisión totalmente consciente, los proyectos en los que el IDT se halla embarcado subrayan el aspecto participativo de la ciudadanía en el sistema jurídico-político.

De hecho, la dimensión democrática que han adoptado progresivamente las aplicaciones de la segunda generación de la WS ha sido reiteradamente señalada por la literatura (e.g. Motta, 2006). El conocimiento se concibe como colectivo, escalable, reutilizable y compartible. Enriquecer con semántica este proceso y concebir servicios jurídicos semánticos (SJS) parece una consecuencia natural de la propia perspectiva de la WS, pero de hecho no resulta tan sencillo. Significa, entre otras cosas: (i) comprender las transformaciones del mercado jurídico, de la sociedad y de la política (es decir, hay que parametrizar las necesidades concretas de los usuarios de la red); (ii) prestar atención a las posibilidades de la nueva generación de aplicaciones de WS (interoperabilidad con los usuarios y las redes sociales); (iii) enfrentarse a los viejos problemas irresueltos, especialmente los que plantean la adquisición de conocimiento y la construcción de ontologías; (iv) y especialmente, empezar por lo más sencillo: de la gestión de la información a la gestión del conocimiento.

Las experiencias previas en ontologías, sistemas de gestión de conocimiento y multimedia han resultado ser de mucha utilidad para la concepción de instrumentos de participación de la ciudadanía en la regulación. Sin embargo, hay un elemento de impredecibilidad que hay que tener muy en cuenta; los ciclos iterativos pueden ser más largos de lo previsto, y el entorno de aplicación de los sistemas puede resultar determinante.

Voy a introducir ahora tres nociones básicas: (i) diálogo en red; (ii) crowdsourcing y (iii) sistema regulatorio. La noción de diálogo tiene aquí un sentido ligeramente distinto al que tiene en teoría de la argumentación. Diálogo significa "conversación" o creación de confianza en línea, porque de hecho es la forma más natural de interacción en la red (Solis, 2009).

Crowdsourcing, más allá de los ejemplos laborales de Mechanical Turk y de MicroWorks, significa resolución de micro-problemas en línea o, más simplemente, producción de conocimiento agregado (para una tipología: Geiger, Seedorf, Schulze y Nickerson, 2011). Es la situación 
producida por la transformación del conocimiento individual en colectivo a partir de la respuesta distribuida de una comunidad o de una población que no tiene en principio por qué estar conectada entre si.

Un sistema regulatorio o "sistema de regulación" tiene lugar al contemplar las dimensiones de regulación social, gobernanza política o administrativa y regulación institucional (jurídica) al mismo tiempo (Casanovas, Magre, Lauroba, Poblet et al., 2010). Los patrones de comportamiento y gestión de conflictos emergen, así, de la interacción entre los diversos actores que participan o tienen interés en un mismo tipo de situaciones o problemas.

Estas tres nociones interactúan en la definición de lo que voy a denominar derecho relacional, es decir, la asignación de expectativas de conducta (asignación de derechos y obligaciones) en función de protocolos de actuación compartidos (Casanovas y Plaza, 2012). Esta noción de derecho no contradice o compite con los sistemas jurídicos positivos convencionales, sino que se superpone a (y se funde con) ellos, al emerger de la interacción entre los usuarios de los sistemas y los protocolos de comportamiento que siguen para realizar sus objetivos.

Creo que todos los sistemas de Online Dispute Resolution (ODR) precisan de este nicho o contexto de actuación (framework) no jurisdiccional para que los acuerdos que constituyen el resultado de la negociación tengan sentido. Este es de hecho el soporte o conjunto de escenarios sociales para que los modelos automatizados de negociación -como los desarrollados sobre el BATNA por John Zeleznikow y Arno Lodder (2010, 146 ss.), u otros basados en modelos narrativos o dialógicos - puedan anclarse y ser contextualmente efectivos (World Wide Web Consortium, 2012).

El derecho relacional, por así decirlo, es el nicho ecológico en el que pueden encapsularse distintos tipos de tecnología que convergen para obtener un resultado común. La condición para su existencia es la capacidad de constituir una comunidad o red social basada en la confianza online que cree un valor agregado mediante su propia interacción. Se trata, en suma, de la emergencia del vínculo existente entre la web 2.0 y 3.0 , entre la web social y la web de datos, a partir de los protocolos de uso de sus usuarios (no importa si son ciudadanos, consumidores, empresas o instituciones). La figura 6 es el diseño ortogonal pensado entre una plataforma de servicios de negociación y mediación online (ONTOMEDIA) y la implementación de instituciones jurídicas electrónicas (Electronic Legal
Institutions) que modelan las fases de los procesos de negociación, conciliación, mediación y arbitraje. La ELI para resolución de disputas en línea (ODR), fue desarrollada por Pablo Noriega (et al.) para el Libro Blanco de la Mediación en Cataluña, y el programa fue incluido como anexo al Cap. 16 de la versión castellana para su uso público (http://llibreblancmediacio.com).

\begin{tabular}{|c|c|c|}
\hline \multicolumn{2}{|c|}{ Electronic Legal Institution (ELI) } & $\mathrm{O}$ \\
\hline Web 3.0 & & $\begin{array}{l}\mathrm{T} \\
\mathrm{O}\end{array}$ \\
\hline \multirow[t]{2}{*}{ Web 2.0} & $\begin{array}{l}\text { Semantic Web } \\
\text { (RDFS, OWL) }\end{array}$ & M \\
\hline & $\begin{array}{l}\text { Linked Data (RDF, } \\
\text { SPARQL) }\end{array}$ & $\begin{array}{l}\mathrm{I} \\
\mathrm{A}\end{array}$ \\
\hline
\end{tabular}

Figura 6. Estructura ortogonal de ELI + ONTOMEDIA, sobre un diseño original de James Hendler (2009). Fuente: Casanovas et al. (2010).

Los ejemplos de este tipo de estructuras se dan también en la actividad de plataformas como Ushahidi (http://ushahidi.com/) o Reforme.ma (http://www.reforme.ma/). Ushahidi ("testimonio" en Swahili) es la plataforma más utilizada por los denominados crisis mappers en los conflictos o crisis humanitarias (como en los casos de Libia, Siria, o Somalia) (Poblet et al., 2011). Reforme.ma es la plataforma para crowdsourcing jurídico-político creada por Tarik NeshNash para monitorizar la elaboración de la Constitución en Marruecos.

\subsection{Web 3.0 y derecho relacional}

La plataforma de servicios jurídicos de mediación que concebimos en ONTOMEDIA ( $\mathrm{y}$ ahora en CONSUMEDIA) intenta facilitar el acceso a la justicia del ciudadano (Poblet et al., 2011). En el proyecto original, se trataba de una plataforma con una doble funcionalidad de servicios: (i) para los mediadores profesionales, (ii) para los ciudadanos, siguiendo la compartimentación de campos que habíamos establecido en el Libro Blanco de la Mediación en Cataluña (2010-11). Con el tiempo, sin embargo, y siguiendo la línea anteriormente trazada, este proyecto se ha convertido en un proyecto de aplicación a tecnología móvil, en el que el diseño original se ha sectorializado de forma que "acompañe" como un instrumento útil al consumidor que pretenda presentar una reclamación. 
Este paso significa profundizar en la gestión de conocimiento. Dicho de otro modo, la simplificación del acceso a la justicia, la capacidad de gestionar los propios conflictos, no significa simplificar la gestión de datos, sino todo lo contrario. Hemos debido construir: (i) una base de datos con más de 30.000 casos de consumo, (ii) tipologías de reclamaciones, (iii) y aplicar clustering estadístico y de NLP para ello (FernándezBarrera y Casanovas, 2011). Hemos proporcionado asimismo al sistema aplicativos para encontrar la agencia de consumo y los procesos de reclamación más próximos al consumidor, mediante otra base relacional construida al efecto (3). Al desarrollo de MediWeb, para PCs, y MediApp para móvil (González-Conejero, Meroño-Peñuela, Suquet y Martínez-Pujol, 2011), ha seguido la construcción de GeoConsum, ya accesible en red (https://play.google.com/store/ apps/details?id=com.idt.ontomedia.geoconsum).

Este proceso es paralelo a la construcción de archivos relacionales inteligentes en open access para agregar y gestionar de forma más centralizada la información, blogs, blawgs, escritos de literatura gris y publicaciones de revista que circulan por la red. Las formas de publicación de contenidos científicos están cambiando aceleradamente (Osman, Sierra, Sabater-Mirar, Wakeling, Simon, Origgi y Casati, 2010; Casati, Casati, Gerstner, Giunchiglia, Marchese, Origgi, Rossi, Sierra y Cheng-Chang, 2011). También los jurídicos (Francesconi y Peruginelli, 2011; Casanovas y Plaza, 2011).

Lo que estamos descubriendo, en suma, es que facilitar el proceso de agregación de conocimiento implica incrementar el trabajo e instrumentos de distintas tecnologías que deben ir por debajo, sin que el usuario las perciba. "Poco a poco - recuerda Schorlemmer (2011) — se está definiendo un espacio global de datos sobre personas, compañías, libros, publicaciones científicas, películas, música, programas de radio y de televisión, genes, proteínas, fármacos y ensayos clínicos, comunidades en línea, datos estadísticos y científicos, etc., que a día de hoy se estima en 32.000 millones de tripletes RDF con 500 millones de enlaces entre ellos".

Pensar más funcionalidades, "empoderar" al usuario para no solamente producir, sino gestionar su propio conocimiento a partir de iniciativas como Open Data Stack (Davies, 2011), Linked Services (Pedrinaci y Domingue, 2011) o Linked Open Legal Data (Casellas y Vallbé, 2012) no significa disminuir, sino aumentar el riesgo de conflictos y situaciones irresueltas (de no-justicia). Esto se aplica a la evolución de las formas de ODR también. Por ejemplo, Colin Rule, que estaba detrás del mecanismo com- pensatorio de e-Bay y PayPal, acaba de lanzar http://modria.com/, una plataforma de ODR para solucionar los conflictos de reputación en el sector de la hostelería: son los propios dueños de hoteles, moteles y restaurantes los que entraban sistemáticamente en los sistemas de rating de la competencia para disminuir su reputación en la red.

El desarrollo del derecho relacional implica y presupone asimismo el desarrollo del conocimiento aplicado al derecho positivo que los modelos de informática jurídica documental de MetaLex y Akoma Ntoso representan. Derecho relacional y autorregulación son cosas distintas y no deben confundirse, como mostramos en el Proyecto de Código de Buenas Prácticas para el Audiovisual de Cataluña (Teodoro, Galera y Casanovas, 2010). Así, los modelos de derecho relacional son necesarios para reconstruir los sistemas regulatorios de ámbitos aparentemente alejados de los acuerdos y del diálogo, como e.g. la prevención de la criminalidad organizada (cfr. CAPER http://www.fp7-caper.eu/fr.html). Lo esencial no son los acuerdos, ni tan siquiera la horizontalidad de las relaciones, sino las distintas dimensiones (social, de gobernanza, jurídica) de un tipo de regulación compleja cuyo procesamiento a través de la red modifica la concepción de protección de los ciudadanos y la gestión de la seguridad de las relaciones sociales. Incluso en este ámbito, antes estatal, cada vez importa más la participación de los propios ciudadanos (afectados en distinto grado), la vecindad digital. Otra vez el problema radica en los medios de agregación y gestión de la información, en la construcción de una racionalidad colectiva y de un conocimiento compartido a partir de las intervenciones singulares.

De este modo, el derecho relacional se añade como una tercera línea a las dos perspectivas respecto al derecho al uso en la literatura e institutos de investigación - derecho de las TICs (IT Law) y tecnología para los profesionales del derecho (IT for Lawyers).

Resulta especialmente relevante darse cuenta de que la red ya ha entrado a formar parte de la vida cotidiana de la ciudadanía, las empresas y la administración. Es urgente volver a plantear los temas de ética aplicada como la confianza, la dignidad y la honestidad no solamente en línea, sino en la continua intersección entre computación y efectos sociales, económicos y políticos. Igualmente es urgente repensar la relación entre democracia y Derecho para plantear jurídicamente las cuestiones de privacidad y propiedad intelectual e intentar dar una salida a los conflictos de patentes. Para ello no basta con el uso convencional de los conceptos de la 
tradición de Derecho público y privado (Roig, 2010). Es necesario pensar en los escenarios sociales que la Web 3.0 y el Linked Open Data suscitan, y volver a reformular de forma integrada el conocimiento jurídico positivo, profesional y relacional. Mejor empezar desde el principio, pues, antes de modelar: a partir de una descripción minuciosa y un conocimiento preciso de los procesos jurídicos y su engarce en escenarios sociales (Roca, 2011).

Buscar lo simple resulta, pues, complejo. Creo que esta paradoja resume bien los retos de las aplicaciones semánticas al Derecho y a la regulación. Lo que suceda ahora va a depender de la evolución y convergencia de tecnologías distintas a la WS, especialmente en el sector de las tablets, tecnología móvil y computación en la nube (cloud computing).

\section{A modo de síntesis}

Se ha ofrecido un rápido panorama de cómo el desarrollo de la nueva generación de servicios web puede incorporarse al mundo jurídico. $\mathrm{He}$ definido tres aproximaciones teóricas distintas a la Web Semántica. Las estrategias de MetaLex (Leibniz Center, Amsterdam) y Akoma Ntoso (CIRSFID, Bolonia) pretenden incidir en la gestión informática documental de los textos jurídicos, ofreciendo servicios de anotación, descripción, clasificación y búsqueda más rápida y eficaz. La estrategia del IDT-UAB (Barcelona) sigue un camino paralelo. No se trata de informática jurídica documental como tal en sentido estricto, sino de desarrollar el modelo democrático de participación de la ciudadanía (o, mejor aún, de la vecindad digital) facilitando el acceso a la justicia, a mecanismos de autorregulación y a formas de crowdsourcing político-jurídico. En el primer caso, la teoría social y jurídica se integra en la ingeniería del conocimiento. En el segundo, es la ingeniería del conocimiento la que se integra en la teoría social y jurídica. Pero en todos ellos es primordial la convergencia de ciencia, tecnologías y regulación.

Sin duda, estas estrategias no agotan el panorama. Me he extendido un poco más de lo debido quizás en la exposición de nuestro propio trabajo. Existen muchas más perspectivas en informática jurídica, como las basadas en las técnicas de lingüística computacional desarrolladas en el ITTIG-CNR de Florencia y en el CERSA-CNR de Paris; o como las aproximaciones a la robótica y a los sistemas multiagente (MAS) de la Universidad de Torino, entre otros centros.

En España, hay institutos importantes como el de IIIA-CSIC de cuyo trabajo hemos aprendido mucho y que últimamente ha planteado proyectos esenciales sobre MAS, ontologías contextuales, inteligencia social y "tecnologías del acuerdo" -cfr. Agreement Technologies, http://www.agreement-technologies.org/, y su desarrollo como COST Action en http://www. agreement-technologies.eu/ (Ossowski, 2012). Creo que, hoy en día, para abordar los temas clásicos de los sistemas normativos o los sistemas de negociación y mediación, resulta ya imprescindible incorporar esta perspectiva a la reflexión sobre el derecho y la regulación.

En el campo estrictamente jurídico, ha habido una especial atención a la educación en línea (la red LEFIS, DerechoTICs, UOC) y hace tiempo que la filosofía jurídica analítica converge con la ingeniería del conocimiento (en las Universidades de Murcia, Zaragoza, Valladolid, entre otras). No me he detenido hoy en ellas, y ciertamente merecería también la pena.

\section{Agradecimientos}

Este artículo es una revisión de la ponencia presentada en el III Simposio de Informática Jurídica Documental, Facultad de Derecho, Universidad de Murcia, 23-24 de Noviembre 2011. He variado ligeramente el título. Agradezco a Rafael Hernández Marín y a Juan-José Iniesta su amable invitación a participar en el Simposio.

ONTOMEDIA (CSO-2008-05536-SOCI; TSI-0205012008-131), SGR (CIRIT-2009SGR0688), CONSUMEDIA INNPACTO IPT-2011-1015-430000, CAPER EU SEC2010.1.2-1. Project 261712, AGREEMENT TECHNOLOGIES EU COST IC0801.

\section{Notas}

(1) La última versión de WordNet es 3.1, de Junio de 2006. La base de datos contiene 155.287 palabras organizadas en 117.659 synsets para un total de 206. 941 pares de sentido. Cfr. Wordnet, Princeton (2006). Los Wordnet en castellano y en catalán se construyeron dentro del marco general de EuroWordnet, Cfr. NLP Research Group UPC (2012).

(2) BATNA es el acrónimo que corresponde a "Best Alternative to a Negotiated Agreement", base del denominado modelo de Harvard.

(3) Esta base de datos desarrollada por el IDT proporciona información sobre la legislación aplicable en el ámbito de consumo en España. Incluye 188 disposiciones legales, de las cuales 49 son de ámbito estatal, 28 son disposiciones de Derecho derivado europeo y 111 disposiciones de ámbito autonómico. Asimismo, la base de datos incluye un repositorio sobre entidades de protección a los consumidores, tanto de ámbito público (Agencias de protección al consumidor de las distintas CCAA, OMICs y OCICs) como de ámbito privado (asociaciones de consumidores). La base de datos incluye 1275 entidades de protección al consumidor de 903 municipios del Estado.

\section{Referencias}

Antoniou, Grigoris; van Harmelen, Frank (2010). Manual de Web Semántica (A Semantic Web Primer, MIT Press, 
2008, $2^{a}$ ed.). // Traducción y edición de Núria Casellas y Manuel Atencia, Colección La Razón Áurea, Granada: Comares.

Baeza-Yates, R. (2009). The Next Web. http://thenext web.com/2009/04/16/ricardo-baezayates/ (04/04/2012).

Barabucci, G.; Cervone, L.; Palmirani, M. Peroni, S.; Vitali, F. (2010). "Multi-layer Markup and Ontological Structures in Akoma Ntoso". // Casanovas, P., G. Sartor. Casellas, R. Rubino (Eds.), Artificial Intelligence and Complex Legal Systems, AICOL Workshops 2009, LNAI 6237, Berlin, Heidelberg: Springer Verlag, 133-149.

Benjamins, R.V.; Casanovas, P.; Breuker, J.; Gangemi, A. (Eds.) (2005). Law and the Semantic Web. Legal Ontologies, Methodologies, Legal Information Retrieval, and Applications. // Lecture Notes in Artificial Intelligence 3369, Springer Verlag: Berlin, Heidelberg

Boer, A. (2009). Legal Theory, Sources of Law, and the Semantic Web. // Frontiers in Artificial Intelligence and Applications, 195, Amsterdam: IOS Press.

Boer, A.; van Engers, T. (2010). Knowledge Acquisition from Sources of Law in Public Administration. // P. Cimiano and H.S. Pinto (Eds.), EKAW2010, LNAI 6317, Heidelberg, Berlin: Springer Verlag, 44-58.

Bourcier, D., Casanovas, P., Dulong de Rosnay, M., Maracke, C. (Eds.) (2010). Intelligent Multimedia. Managing Creative Works in a Digital World. // Florence: European Press Academic Publishing.

Bratt, Steve (2006). Data on the Web. http://www. w3.org/2006/Talks/1023-sb-W3CTechSemWeb/W3CTec hSemWeb.pdf (02/04/2012).

Breuker, J.; Boer, A.; di Bello, Boer, A. (2007). The LKIF Core Ontology of Basic Legal Concepts. // Casanovas, P., M.A. Biasiotti, E. Francesconi, M. T. Sagri (Eds.) Proceedings of LOAIT '07II Workshop on Legal Ontologies and Artificial Intelligence Techniques. 43-64. http://www.ittig.cnr.it/loait/LOAIT07-Proceedings (04/04/2012)

Brodkin, J. (2007). Gartner touts Web 2.0, scoffs at sequel. Web 3.0 just a marketing ploy, but collaborative tools are here to stay. // Networkworld. http://www.networkworld. com/news/2007/092107-gartner-web-20.html?page=2 $(2 / 04 / 2012)$

Bruce, T. R. (2000). Tears Shed Over Peer Gynt's Onion: Some Thoughts on the Constitution of Public Legal Information Providers. // Journal of Information, Law \& Technology. 2. http://www2.warwick.ac.uk/fac/soc/law/ elj/jilt/2000_2/bruce/ (13/06/2011).

Bruce, T. R. (2009). Foundings on the Cathedral Steps. // Peruginelli, G.; Ragona M. (Eds.). Law via the Internet. Free Access, Quality of Information, Effectiveness of Rights, Florence: European Press Academic Publishing. 441-422.

Casanovas, P. (2010). Legal Electronic Institutions and ONTOMEDIA: Dialogue, Inventio, and Relational Justice Scenarios. // Casanovas, P.; Sartor, G.; Pagallo, U.; Ajani, G. Al Approaches to the Complexity of Legal Systems (AICOL I-II). The Semantic Web, multilingual ontologies, multiagent systems, distributed networks, LNAI 6237, Heidelberg, Berlin: Springer Verlag. 184-204.

Casanovas, P. (2012). Crowdsourcing and relational law: A preliminary approach. // Actes del II Congrés Català de Filosofia, Sueca, 9th -11th 2011, València: Publicacions de la Universitat de València (en prensa).

Casanovas, P.; Poblet, M. (2008). Concepts and Models of Relational Justice. // Casanovas, P.; Sartor, G.; Pagallo, U.; Ajani, G. Al Approaches to the Complexity of Legal Systems (AICOL I-II). The Semantic Web, multilingual ontologies, multiagent systems, distributed networks, LNAI 6237, Heidelberg, Berlin: Springer Verlag. 323339.
Casanovas, P.; Poblet, M. (2009a). Justice via the Internet: Hopes and Challenges of Law and the Semantic Web. // G. Peruginelli; Ragona, M. (eds.). Law via the Internet. Free Access, Quality of Information, Effectiveness of Rights. Florence: European Press Academic Publishing. 347-359. (Series in Legal Information and Communication Technologies, 5).

Casanovas, P.; Poblet, M. (2009b). Justicia relacional, servicios web y la segunda generación de la web semántica. // F. Galindo y A.J. Rover (Eds.). Derecho, gobernanza y tecnología: materiales para el aprendizaje del Derecho en la sociedad del conocimiento. Zaragoza: Prensas Universitarias de Zaragoza. 245-276. (Serie LEFIS, 7).

Casanovas, P.; Binefa, X.; Gracia, C.; Teodoro, E.; Galera, N.; Blázquez, M.; Poblet, M.; Carrabina, J.; Montón, M.; Montero, M; J. Serrano; López-Cobo, M. (2009). The esentencias Prototype: a Procedural Ontology for Legal Multimedia Applications in the Spanish Civil Courts. // Breuker, J.; Casanovas, P.; Klein, M. C. A.; Francesconi, E. (eds.). Law, Ontologies and the Semantic Web: Channelling the Legal Information Flood., , Amsterdam, Berlin: IOS Press. 199-219. (Frontiers in artificial Intelligence and Applications, 188).

Casanovas, P.; Magre, J.; Lauroba, E.; Díaz, L.; Poblet, M.; et al. (2010). Introducción: marco conceptual, metodología i guía de lectura. // Generalitat de Catalunya, Departament de Justícia. Libro Blanco de mediación de Cataluña. Barcelona: Huygens Editorial. 29-82.

Casanovas, P.; Casellas, N.; Vallbé, J. J. (2011) EmpiricallyGrounded Development of Legal Ontologies: a SocioLegal Perspective. // Sartor, G.; Casanovas, P.; Biasotti, M. A.; Fernández-Barrera, M. (eds.). Approaches to Legal Ontologies. Theories, Domains, Methodologies. Dordrecht, Heidelberg, London, New York: Springer Verlag. 49-68. (Law, Governance and Technology Series, 1).

Casanovas, P.; Plaza, E. (2012). Advancing an Open Access Publication Model for Legal Information Institutes. // AICOL-3, XXV IVR World Conference in Social Philosophy and Philosophy of Law, Frankfurt 15th-20th August 2011, LNAI. Springer Verlag (en prensa).

Casellas, N. (2011). Legal Ontology Engineering: Methodologies, Modelling Trends, and the Ontology of Professional Judicial Knowledge. Heidelberg, Berlin: Springer Verlag. (Law, Governance and Technology Series. 3).

Casellas, N.; Vallbé, J.J. (2012). Legal Information and Linked Open Legal Data. X. Serra (Ed.), Actes del II Congrés Català de Filosofia (Sueca, 9-11 Novembre 2011), València: Publicacions de la Universitat de València (en prensa).

CommonKADS (2000). http://www.commonkads.uva.nl/ (01/04/2012).

Casati, F.; Casati, R.; Gerstner, R.; Fausto Giunchiglia, F., Marchese, M.; Origgi, G.; Rossi, A.; Sierra, C., ChengChang, Yi (s. d.). D6.1v1 Innovative publisher services for liquid publications, https://dev.liquidpub.org/svn/liquid pub/papers/deliverables/LP_D6.1v1.pdf (05/04/2012).

D’Aquin, M., Motta, E.; Sabou, M.; Angeletou, S.; Gridinoc, L.; López, V.; Guidi, D. (2008). Toward a New Generation of Semantic Web Applications. // IEEE Intelligent Systems. (May-June 2008) 20-28.

Davies, J.; Potter, M.; Richardson, M.; Stinčić, S.; Domingue, J.; González-Cabero, G. (2009). Towards the Open Service Web. // BT Technology Journal. 26:2. http://www. btplc.com/innovation/journal/BTTJ/current/HTMLArticles/ Volume26/25Towards/Default.aspx (07/04/2012).

Davies, T. (2011). Open Data Impacts. What's in the Linked Open Data Stack? Davies on May 2, 2011. // Update: Parts of the Linked Open Data Puzzle (15.00, May 2nd 
2011) http://www.opendataimpacts.net/page/2/ (02/04/2012).

Fernández-Barrera, M.; Casanovas, P. (2011). Towards the intelligent processing of non-expert generated content: Mapping web 2.0 data with ontologies in the domain of consumer mediation. // AHLTL, ICAIL, Pittsburgh, 10 junio 2011. http://wyner.info/LanguageLogicLawSoftwa re/index.php/2011/01/29/icail-workshop-applying-humanlanguage-technology-to-the-law/ (06/04/2011).

Francesconi, E.; Peruginelli, G. (2011). An Open Access Policy for Legal Informatics Scholarly Research Publishing, AICOL III, IVR-XXV World Conference in Philosophy of Law and Social Philosophy, LNAI, Berlin. Heidelberg: Springer Verlag (en prensa).

Geiger, D.; Seedorf, S.; Schulze, T; Nickerson, R. (2011). Managing the Crowd: Towards a Taxonomy of Crowdsourcing Processes. // AMCIS-Proceedings of the Seventeenth Americas Conference on Information Systems, Detroit, Michigan August 4th-7th 2011.

GeoConsum. IDT, 2011. https://play.google.com/store/apps/ details?id=com.idt.ontomedia.geoconsum.

González-Conejero, J.; Meroño-Peñuela, A.; Suquet, J.; Martínez-Pujol, O. (2011). Online Mediation Consumer Tools: MediWeb and MediApp, e Paul Cunningham and Miriam Cunningham (Eds), e-Challenges e-2011 Conference Proceedings, http://www.echallenges.org/e2011/ outbox/eChallenges_e20011_ref_128_doc_7435.pdf (06/04/2011)

Greenleaf, G. (2010). The global development of free access to legal information. // A. Paliwala (Ed.). A history of legal informatics. Zaragoza: Prensas Universitarias de Zaragoza, LEFIS.

Hoekstra, Rinke (2011). The MetaLex Document Server Legal Documents as Versioned Linked Data. // Proceedings of the 10th International Semantic Web Conference, ISWC 2011.

Howe, J. (2006). The Rise of Crowdsourcing. // Wired. (14 June). http://www.wired.com/wired/archive/14.06/crowds .html (29/04/2011).

Lodder, A.; Zeleznikow, J. (2010). Enhanced Dispute Resolution Through the Use of Information Technology. Cambridge: Cambridge University Press.

META-LEX. (2006). CEN MetaLexWorkshop Agreement. http://svn.leibnizcenter.org/svn/MetaLexWS/documentati on/2006/agreement-20061206.html (01/04/2012).

Motta, E. (2006). Knowledge Publishing and Access on the Semantic Web: A Sociotechnological Analysis. // IEEE Intelligent Systems, May/June, 2006, pp. 88-90.

NLP Reserch Group (2012). http://nlp.Isi.upc.edu/ web/index.php?option=com content\&task=view\&id=31\&l temid=57 (01/04/2012).

Noriega, P.; López de Toro, C.; Montero, R.; Pérez Martínez, H. (2010). Anexo: Prototipo de un Sistema Genérico de Apoyo a la Mediación. // LBMC. Cap. 16. 985-1006. http://llibreblancmediacio.com (06/04/2012).

Osman, N.; Sierra, C.; Sabater-Mir, J.; Wakeling, J. R.; Simon, J.; Origgi, G.; Casati, R. (2010). Liquid Publications and its Technical and Legal Challenges. // Bourcier, D.; Caanovas, P.; Dulong de Rosnay, M.; Maracke, C. Intelligent Multimedia: Managing Creative Works in a Digital World. Florence: European Press Academic Publishing. 321-336.

Ossowski, S. (Ed) (2012). Agreement Technologies. Heidelberg, Berlin: Springer Verlag (Law, Governance and Technology Series, 8).

Palmirani, M.; Cervone, L.; Vitali, F. (2011). A Legal Document Ontology: The Missing Layer in Legal Document Modelling. // G. Sartor; Casanovas, P.; Biasiotti, M. A.; Fernández-Barrera, M. (eds.), Approaches to Legal On- tologies: Theories, Domains, Methodologies. Dordrecht, Heidelberg: Springer Verlag. 167-200. (Law, Governance and Technology Series. 1).

Pedrinaci, C.; Domingue, J. (2010). Toward the Next Wave of Services: Linked Services for the Web of Data. // Journal of Universal Computer Science. 16:3 (2010) 1694-1719.

Poblet. M. (2011). Móviles, mapas, satélites y redes sociales: gestión de crisis 2.0. // Lychnos: Cuadernos de la Fundación General del CSIC. 7. http://www.fgcsic. es/lychnos/es_ES/articulos/ (05/04/2012).

Poblet, M.; Casanovas, P.; López-Cobo, J.L.; Casellas. (2011). ODR, Ontologies and Web 2.0. // Journal of Universal Computer Science, J.UCS, vol. 17. 4 (2011): 618634.

Poulin, D. (2009) Fifteen Years of Free Access to Law. // Peruginelli, G. and Ragona M. (Eds.), Law via the Internet: Free Access, Quality of Information, Effectiveness of Rights. Florence: European Press Academic Publishing. 15-32.

Roca, M.; Serra, X. (ed.). Mobiliser le droit, se saisir du droit et faire réaliser le droit. Sites de mobilisations collectives des associations homosexuelles en Suisse et en Espagne. // Actes del II Congrés Català de Filosofia (Sueca, 9-11 Novembre 2011). València: Publicacions de la Universitat de València (en prensa).

Roig, A. (2010). Privacy and Social Networks: From data Protection to Pervasive Computing. // Genesereth, M., Vogl, R., and M.A. Williams (Cochairs), Intelligent Privacy Management. Papers from the AAAI Spring Symposium. California: AAAI Menlo Press. 146-149. Technical Report SS-10-05.

Sartor, G.; Palmirani, M.; Francesconi, E; Biasiotti, M.A (eds.) (2011). Legislative XML for the Semantic Web. // Law, Governance and Technology Series. 4. Dordrecht, Heidelberg: Springer Verlag.

Sartor, G. (2011). Legislative Information and the Web. // G. Sartor et al. (Eds.) Legislatiove XML for the Semantic Web. LGTS, 4, Dordrecht, Heidelberg: Springer Verlag. 11-34.

Schorlemmer, M. (2011). Diez años construyendo una web semántica. Lychnos. 7. CSIC. http://www.fgcsic.es/l ychnos/es_ES/articulos/construyendo_una_web_seman tica $(05 / 05 / 2012)$.

Schreiber, A. T.; Akkermans, J. M.; Anjewierden, A. A.; de Hoog. R.; Shadbolt, N. R.; van de Velde, W.; Wielinga, B. J. (2000). Knowledge Engineering and management. The CommonKADS Methodology. Cambridge, Mass.: The MIT Press.

Solis, B. (2009). Conversation prism. http://www.briansolis. com/2009/03/conversation-prism-v20/ (06/04/2012).

Spivak, N. (2007). Gartner is wrong. http://novaspivack. typepad.com/nova_spivacks_weblog/2007/09/gartner-iswron.html (02/04/2012).

Teodoro, E.; Galera, N.; Casanovas, P. (2010). Selfregulation and Policy in the Spanish Audiovisual Sector: The Catalan Code of Best Practices. // Genesereth, M.; Vogel, R.; Williams, A. A. (Cochairs) 2010 AAAI Proceedings Privacy 2010: Intelligent Information Privacy Management Symposium, Stanford-CA. http://www.aaai. org/Press/Reports/Symposia/Spring/spring-reports.php\# SS10.

Vallbé, J. J. (2009). Models of Decision-Making: Facing Uncertainty in Spanish Judicial Settings. Tesis Doctoral. Barcelona: Universidad de Barcelona. http://db.tt/SIbfv Npj (04/04/2012).

Vallbé, J.J.; Fernández-Barrera, M.; Casellas, N.; Casanovas, P. (2012). Lecturas sobre Web Semántica y ontologías jurídicas. Aplicaciones para el derecho en la 
nueva generación de la red. Granada: Ed. Comares.

(Colección La Razón Áurea. Serie Investigación, 4).

Wordnet, Princeton (2006). http://wordnet.princeton.edu/ wordnet/man/wnstats.7WN.html (01/04/ 2012).

Enviado: 2012-04-12.

Aceptado: 2012-06-29. 